\title{
Ein elektrisches Online-Sensorsystem zur kontinuierlichen Messung von Fremdstoffen, chemischen Verbindungen und Wasser in Ölen
}

\author{
Dipl.-Ing. Manfred R. Mauntz; Priv.-Doz. Dr. rer. nat. habil. Jürgen Gegner*; Dipl.-Ing. (FH) Stefan \\ Klingauf**; Prof. Dr.-Ing. Ulrich Kuipers ${ }^{* * * *}$ \\ *, ${ }^{* * *} \mathrm{Cmc}$ Instruments $\mathrm{GmbH}$, Hauptstraße 388, 65760 Eschborn; ** University of Siegen, Paul-Bonatz- \\ Straße 9-11, 57076 Siegen; ${ }^{* * *}$ South Westphalia University of Applied Sciences, Haldener Straße 182, \\ 58095 Hagen, * 06173-320078, mrm@cmc-instruments.de; ** 0271-7404657, juergen.gegner@uni- \\ siegen.de; ${ }^{* * *}$ 06173-320078, sk@cmc-instruments.de, ${ }^{* \star \star *}$ 02331-9330817, kuipers@fh-swf.de;
}

\section{Kurzfassung}

Es wird ein Ölsensorsystem zur kontinuierlichen online Messung der ölqualitätsabhängigen Parameter Leitfähigkeit $k$ und Dielektrizitätszahl $e_{r}$ von Ölen entwickelt. Mit dem als OilQSens ${ }^{\circledR}$ bezeichneten Sensorsystem und dem Ansatz einer Messung der Veränderung des Systems Öl-Maschine soll eine quasi kontinuierliche Bewertung des Maschinen- und Ölverschleißes ermöglicht und vorbeugende Wartungsmaßnahmen indiziert werden. Die Anwendung des Sensorsystems ermöglicht eine Erhöhung der Betriebssicherheit, Standzeiten werden vergrößert, Stillstandzeiten reduziert, Ölwechselintervalle dem tatsächlichen Bedarf angepasst und eine verbesserte ökonomische und ökologische Effizienz erreicht. Beispielanwendungen sind Getriebe, Turbinen, Windenergieanlagen, Hydraulikanlagen, Transformatoren und Motoren.

\section{Einleitung}

Es ist bekannt, dass die jährlichen Verluste durch Reibung und Verschleiß in den Industrieländern ca. 5\% des Bruttosozialprodukts betragen. Für Deutschland errechnet sich eine Summe von etwa $35 \mathrm{G} €$ pro Jahr. Die Gesellschaft für Tribologie (GFT) geht davon aus, dass hier ein Einsparpotenzial von rund5 G€, in etwa von 15\% besteht [1]. Mit Hilfe der entwickelten Sensoren soll ein Beitrag zur Umsetzung dieser Vorgabe und der ökonomischen und ökologischen Energieeffizienz von Maschinen und Anlagen geleistet werden.

Das Hochleistungssensorsystem OilQSens ${ }^{\circledR}$ für die online-Ölzustandsüberwachung ermöglicht neben der verlässlichen Detektion beginnender Schädigungen auch die frühzeitige Bestimmung kritischer Schmierungsbedingungen von Wälzlagern und anderen Getriebeelementen. Es werden Komponenten der spezifischen komplexen Impedanz der Schmieröle in Echtzeit gemessen. Verschleiß der Lager an berührenden Kontaktflächen, Metallabrieb, Fremdteilchen und Schwebstoffe sowie chemisch gebrochene Ölmoleküle, sich bildende Säuren oder Seifen führen zu einer Erhöhung der elektrischen Leitfähigkeit. Diese Zunahme korreliert direkt mit dem Verschmutzungsgrad des Schmierstoffs. Bei den üblichen additivierten Ölen kann über eine zusätzliche simultane hochgenaue Messung der Dielektrizitätszahl der Abbau der Additive detektiert werden. Durch die kontinuierliche Bewertung der chemischen Alterung des Schmierstoffs im Betrieb erschließt sich ein Verfahren zur Verhütung von vorzeitigen, unter Schwingungsbeanspruchung auftretenden Lagerausfällen in Windenergieanlagen durch rechtzeitige Durchführung geeigneter Korrekturmaßnahmen (z. B. Nachadditivieren, Ölwechsel), das über die bloße Früherkennung entstandener irreversibler Laufbahnschäden hinausgeht. Die Änderungen der Dielektrizitätszahl erlauben bei nicht additivierten Schmierölen die Ermittlung des Wassergehalts. Die Messsignale können Web-basiert via LAN oder WLAN an ein Condition-Monitoring-System oder über eine serielle Schnittstelle übertragen werden.

Die hohe Messgeschwindigkeit des OilQSens ${ }^{\circledR}$ ermöglicht eine quasi kontinuierliche Überwachung, präventive zustandsorientierte Wartung und Verschleißbegrenzungsreglungen (auch) von Windturbinen. Diese Online-Kontrolle bewirkt eine Reduktion von Stillstandzeiten und eine Erhöhung der Gesamtlebensdauer.

Im neuen Konzept folgen die Bestimmung der Verschmutzung bzw. Abnahme der Qualität der Schmierstoffe und die quasikontinuierliche Bewertung des Bauteilverschleißes und der Ölalterung dem ganz- 
heitlichen Ansatz einer Echtzeit-Überwachung der Zustandsveränderung des Systems Öl-Maschine. Der Basissensor misst Komponenten der spezifischen komplexen Impedanz der Öle: Beispielsweise bewirken Metallabrieb, gebrochene Ölmoleküle, Säuren oder Ölseifen eine Erhöhung der elektrischen Leitfähigkeit, die wegen des gegenüber all diesen Kontaminationsprodukten geringen Reinwerts des Öls mit dem Verschmutzungsgrad direkt korreliert ist. Die dielektrischen Eigenschaften der Öle werden insbesondere durch den Wassergehalt bestimmt, der deshalb bei nicht additivierten Produkten ebenfalls über eine zusätzliche genaue Messung der Dielektrizitätszahl zugänglich wird. Bei additivierten Ölen lassen sich aus Dielektrizitätszahländerungen auch Aussagen über den Abbau von Additiven ableiten. Für eine effiziente Maschinenausnutzung und treffsichere Schadensverhütung besteht demnach mit dem neuen OilQSens ${ }^{\circledR}$ Ölsensorsystem die Möglichkeit, vorbeugende Wartungsmaßnahmen statt in starren Inspektionsintervallen, zeitgerecht und bedarfsabhängig durchzuführen. Verschmutzungsgrad, ÖlAlterung und -versauerung, Wassergehalt bzw. Zersetzungszustand von Additiven bilden ein aussagefähiges Maß der Schmierstoff- und Werkstoffbeanspruchung für die Onlinebetriebsüberwachung von Bauteilen [2], wie Wälzlagern oder Zahnrädern. Wichtige praktische Beispiele stellen hier Windkraftanlagen, Transformatoren und Motoren dar.

\section{Prinzip des OilQSens ${ }^{\circledR}$-Ölsensorsystems}

Mit dem OilQSens ${ }^{\circledR}$ werden Komponenten der komplexen Impedanzen X der Öle, insbesondere die spezifische elektrische Leitfähigkeit $k$ und die Dielektrizitätszahl $e_{r}$ sowie die Öltemperatur $T$ gemessen. Die Bestimmung der Werte von $\mathrm{k}$ und $e_{\mathrm{r}}$ erfolgt dabei unabhängig voneinander[3] [4].

Der extrem hochohmige Basissensor erfordert ein elektronisches Messverfahren mit einer sehr hohen Störfestigkeit. Das entwickelte elektronische Messverfahren arbeitet mit getakteten, mittelwertfreien Testsignalen.Die Messdatenausgabe erfolgt wahlweise über LAN, WLAN oder eine serielle Schnittstelle.

Der aktive Basissensorteil besteht hier aus zwei oder mehreren Basissensorplatten, die in einem konstanten Abstand zueinander an Metallstiften einer Glas/Metall-Durchführung befestigt sind. Die Platten des Basissensors sind mittig in der Messkammer platziert, so dass eine günstige Anströmung des durchfließenden Mediums gewährleistet wird. Eine spezielle Ausrichtung der Sensorgehäuseteile ist bei dieser Konstruktion nicht erforderlich. Die Materialien des Sensorgehäuses und der Stifte der Glas/MetallDurchführung sind in ihren Ausdehnungseigenschaften genau an die Materialeigenschaften des verarbeiten Glases der Durchführung angepasst. Die Druckfestigkeit ist größer als 100 bar. Bild 1 zeigt eine Skizze des Basissensors.

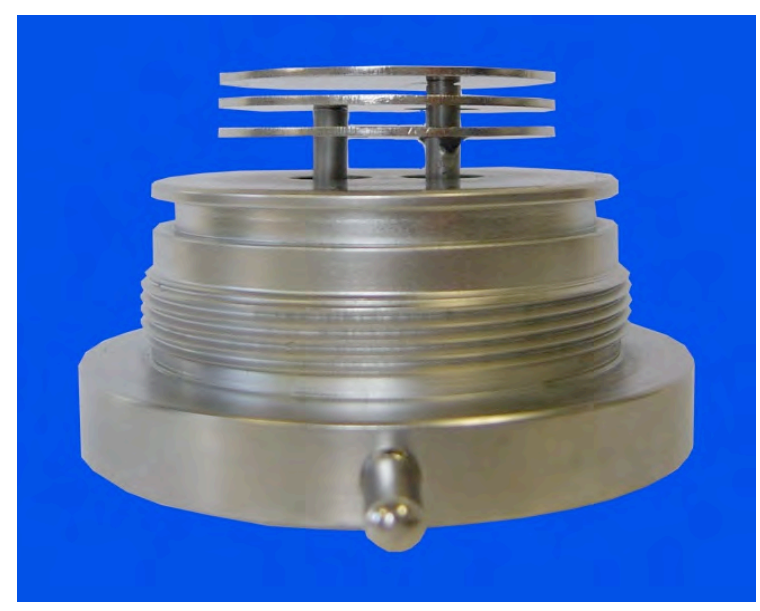

Bild 1: Basissensor mit Drei-Platten-Anordnung. 


\section{Temperaturkompensation und Messgenauigkeit}

Die zusätzliche Messung der Temperatur ist notwendig, da die elektrische Leitfähigkeit und die Dielektrizitätszahl des Öls temperaturabhängig sind. Die Ionen Mobilität und damit die elektrische Leitfähigkeit k sind abhängig von der inneren Reibung des Öls und damit auch von der Temperatur T. Die Leitfähigkeit k des Öls steigt mit der Temperatur T. Die Art der Verschmutzung und ihre Temperaturabhängigkeit kann nicht als bekannt vorausgesetzt werden. Zur Verbesserung der Vergleichbarkeit der Messwerte wurde ein adaptiver Temperaturkompensationsalgorithmus entwickelt. InBild 2 ist die Wirkung der Temperaturkompensation dargestellt.

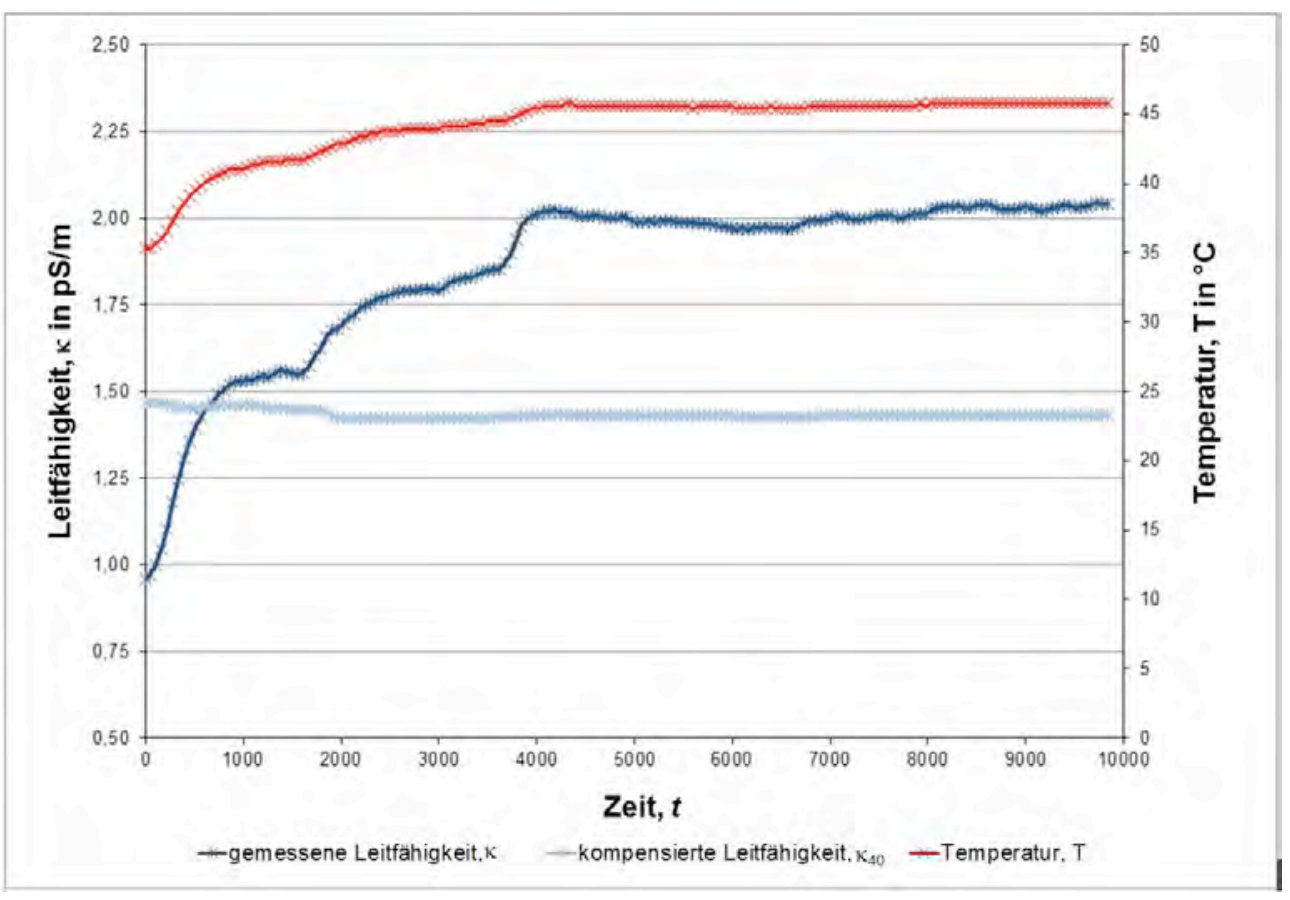

Bild 2: Temperaturkompensationsalgorithmus.

Berechnung der Dielektrizitätszahl und der Leitfähigkeit nach Physikalische Theorienzur Bestimmung der Leitfähigkeit $\mathrm{k}$ und der Dielektrizitätszahl $\mathrm{e}_{\mathrm{r}}$, aus ihren physikalischen Konstanten mit anschließendem Vergleich der gemessenen Werte nach Piekara[5] zeigen bei der Dielektrizitätszahl beispielhaft zwischen den gemessenen und den berechneten Werten Abweichungen kleiner als $2 \%$. Bild 3 zeigt die Abweichungen zwischen den gemessenen und den berechneten Dielektrizitätszahlwerten in \%. 


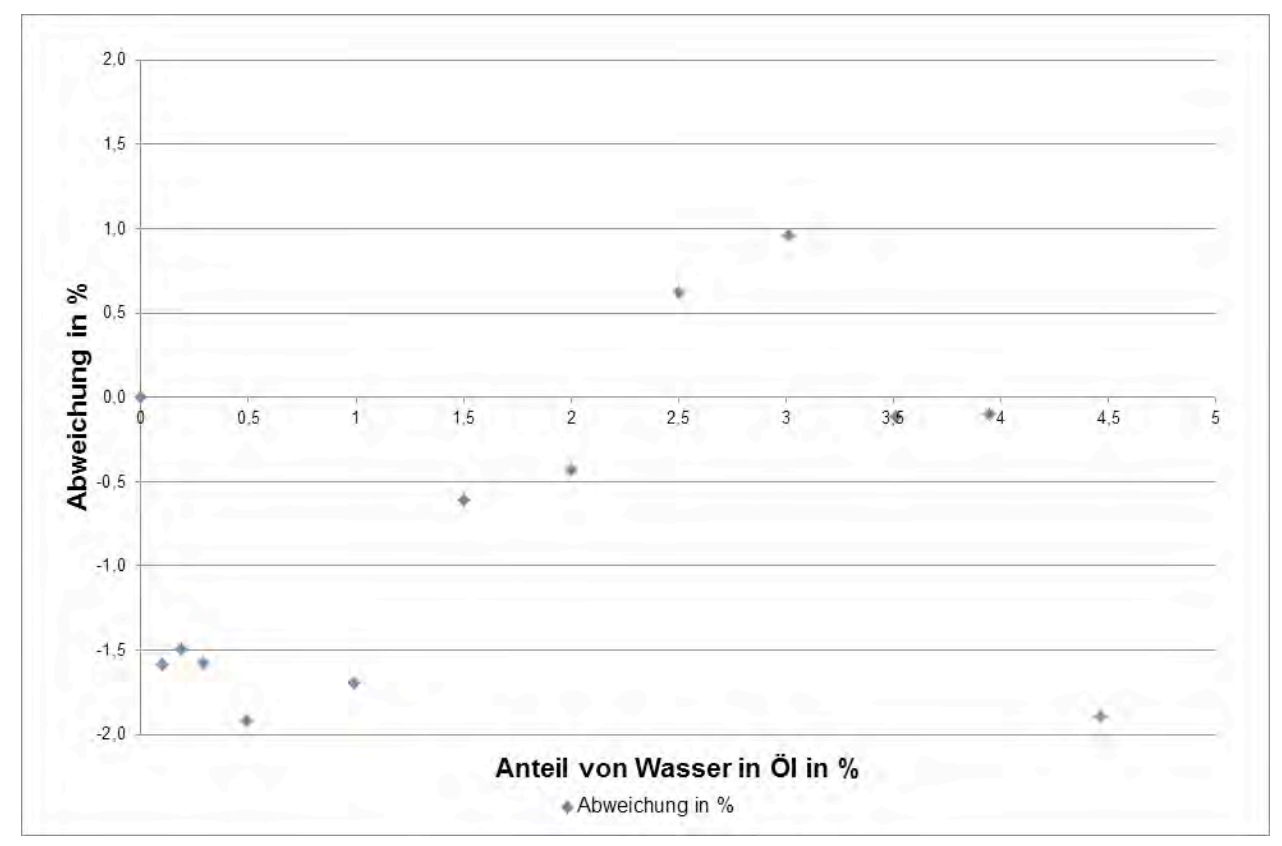

Bild 3: Abweichungen zwischen den gemessenen und den berechneten Dielektrizitätszahlwerten.

\section{Versuch am Wälzlagerprüfstand}

Auf einem Lager- und Getriebeprüfstand der Universität Kaiserslautern werden unterschiedliche Belastungszyklen gefahren und dabei Drehzahlen und Drehmomente gemessen. Das Ergebnis des Versuchs wird im Folgenden mitgeteilt, ausgewertet und diskutiert.

Die drehzahlabhängige Leistung $P(n)$ des Prüfstandes kann wie folgt angegeben werden:

$P=M \cdot \omega, \quad$ mit: $\omega=2 \pi \cdot n$

Hierbei bezeichnet $M$ das Drehmoment, $\omega$ und $n$ symbolisieren die Winkelgeschwindigkeit bzw. die Drehzahl. Mit dem Übersetzungsverhältnis $N_{u}$ ergibt sich die im Getriebe umgesetzte Verlustleistung $\Delta P$ :

$\Delta P=\omega_{1} \cdot\left(M_{1}-N_{\ddot{U}} \cdot M_{2}\right)$

$M_{1}$ und $M_{2}$ bezeichnen das Drehmoment des Antriebs bzw. der Belastung. Im ersten Versuch auf dem Lagerprüfstand wird gezielt ein Lagerschaden erzeugt.

\subsection{Leitfähigkeit des Getrieböls}

Während des Versuchs ändert sich die Leitfähigkeit $\kappa$ des Getriebeöls. Ursache der Leitfähigkeitserhöhung sind neben Temperaturänderungen z. B. abgetragene Verschleißpartikel, Verschmutzungsprodukte, gebrochene Ölmoleküle oder sich bildende Ölseifen. Die Temperaturabhängigkeit der elektrischen Leitfähigkeit des verwendeten Getriebeöls wird kompensiert und eine Umrechnung der jeweils im Getriebeversuch gemessenen Ölleitfähigkeit auf den entsprechenden Leitfähigkeitswert bei $40{ }^{\circ} \mathrm{C}$ durchgeführt.

Bei der zunächst geringen Belastung steigt die elektrische Leitfähigkeit linear mit der Zeit. Vermutlich wächst der in diesem Bereich geringe Lagerverschleiß ebenfalls zeitproportional.Während der notwendigen Abschaltung und dem Hochfahren der Antriebsmaschine auf $330 \mathrm{Nm}$ ist die Leitfähigkeit praktisch konstant. Nach dem Umschalten auf die höhere Last nimmt die Ölleitfähigkeit stark zu. Das Einlaufen (Einspielen) der Lager zeigt sich hier in einer Verminderung der Steigerung der Leitfähigkeit. Ab ca. 30 Minuten vor der Zwangsabschaltung des Versuchs durch einen Schwingungssensor bleibt die Leitfähigkeit näherungsweise konstant, bevor unmittelbar vor der Abschaltung noch ein kurzzeitiger 
Anstieg erfolgt. Nach der Abschaltung des Prüfgetriebes sinkt die Ölleitfähigkeit stark ab. Hier zeigt sich deutlich die Wirkung der Additive: Während der Belastungsphasen werden mehr Verschmutzungsprodukte pro Zeiteinheit erzeugt als über Additive gebunden werden können. Nach der Abschaltung des Prüfstands fällt eine solche Ölkontamination nicht mehr an, wobei die Wirkung der Additive aber fortdauert.

In Bild4 sind die Änderungen der elektrischen Leitfähigkeit aufgetragen. Die Darstellung gibt dabei jeweils über 3 Minuten Intervalle berechnete Mittelwerte wieder.

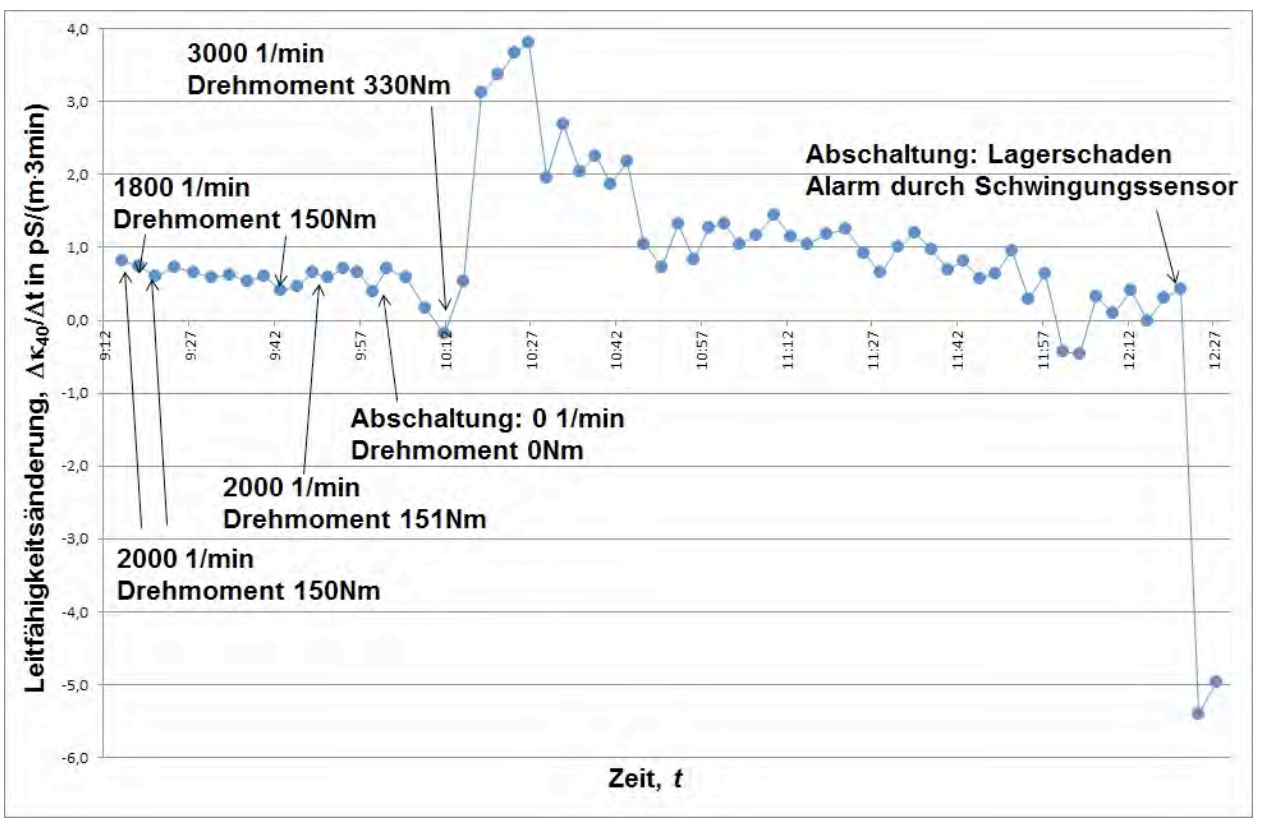

Bild4: Änderung der Leitfähigkeit mit der Prüfzeit, ausgedrückt als $\Delta \kappa_{40} / \Delta \mathrm{t}$.

Beim Anfahren mit 2000 Umdrehungen pro Minute und einem Drehmoment von $150 \mathrm{Nm}$ zeigt sich eine relativ konstante Leitfähigkeitsänderung von 0,6 bis $0,8 \mathrm{pS} / \mathrm{m} \cdot 3 \mathrm{~min}(3,3$ bis $4,4 \mathrm{fS} / \mathrm{m} \mathrm{s})$. Nach der Änderung des Drehmoments auf 330Nm und der Drehzahl auf $3000 \mathrm{~min}^{-1}$ steigt die Leitfähigkeitsänderung stark an, dieses lässt auf die Bildung von ersten Haarrissen schließen [6]. Bild 5zeigt Haarrisse wie sie typischer Weise in Rollenlagern als Beginn der Schädigungen auftreten.

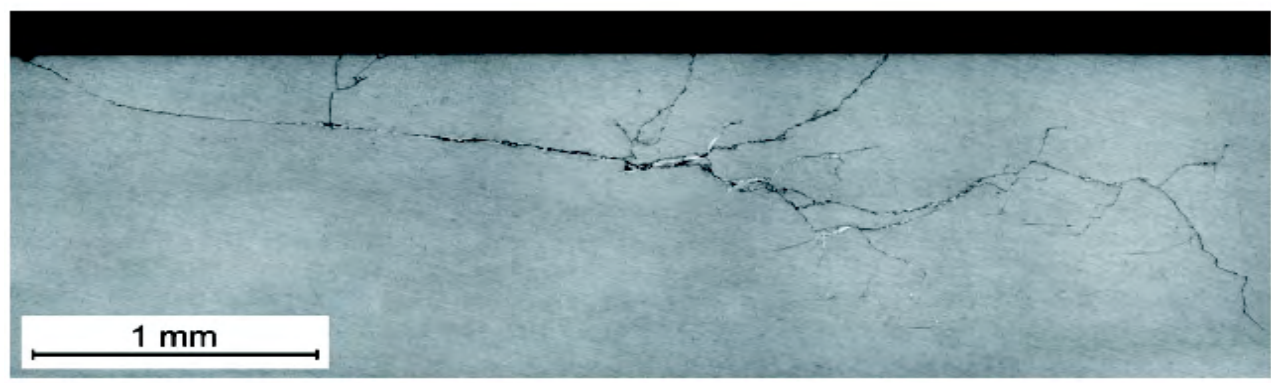

Bild 5: typische Haarrisstruktur[6]

Mittelfristig ist eine Versprödung durch Wasserstoffaufnahme in Metallen mit Gitterdefekten denkbar [7] [8]. Bild 6zeigt hohe interkristalline Sprödbruchanteile, was auf Wasserstoffversprödung durch eingedrungene Schmierstoffbestandteile hinweist. 


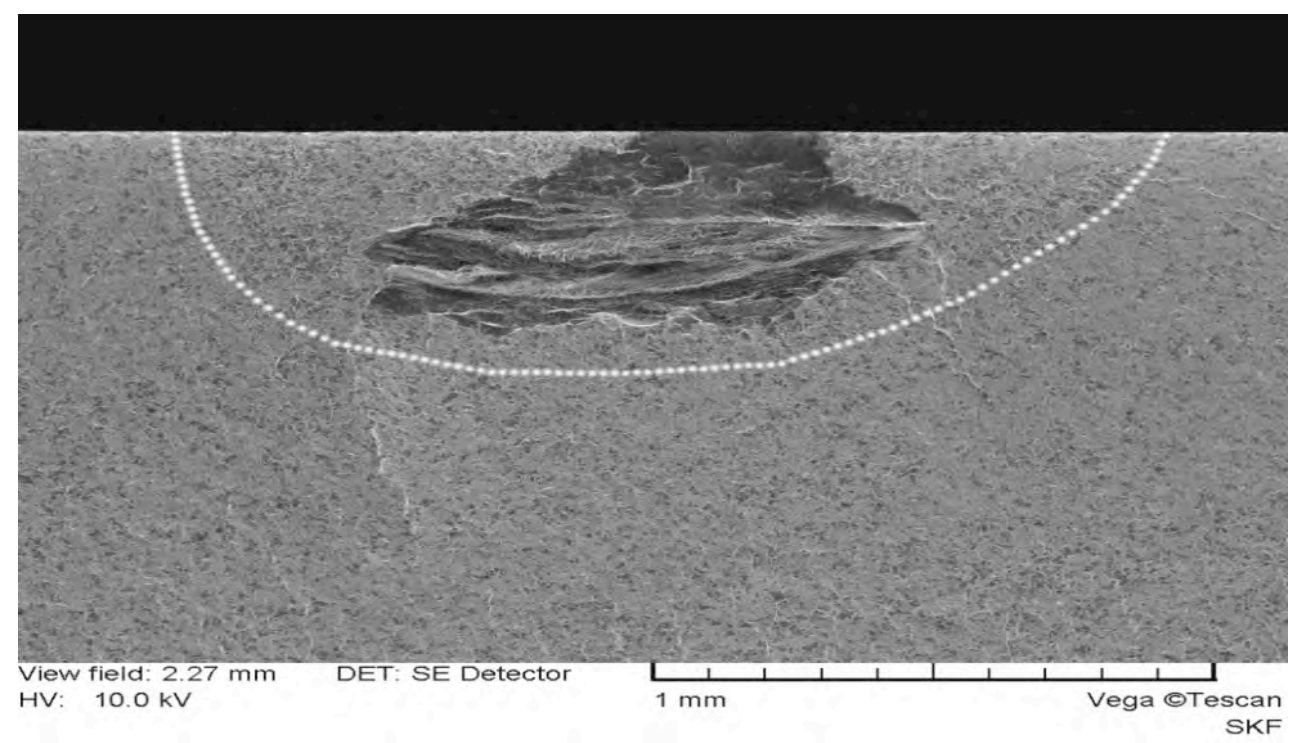

Bild 6: durch Wasserstoffversprödung verursachte interkristalline Sprödbruchanteile [9]

Dies ist die Wirkung der Schwingungskorrosion. DasBild 6zeigt hohe interkristalline Sprödbruchanteile.Es liegt eher eine Werkstoffbeanspruchung und Werkstoffversagen bei zyklischer Wälzbelastung, insbesondere eine oberflächennahe Schädigung von Wälzlagern aus dem Tribokontakt vor [10][11]. Nach der Belastungserhöhung zeigt sich eine steigende Dynamik (Spitzen) in der Änderung der Ölleitfähigkeit. Dies lässt auf zeitlich schwankenden Verschmutzungsanfall und Veränderungen der Lagerbelastungen schließen, wie es beim Bilden von Pittings zu erwarten ist [12]. Die Sprünge in der Steigung der elektrischen Leitfähigkeit vor dem Abschalten sind ein Indiz für den endgültigen Lagerschaden. Bild 7zeigt den Innenring des ausgefallenen Planetenradlagers mit massiver Laufbahnschädigung nach Ende des Versuchs.

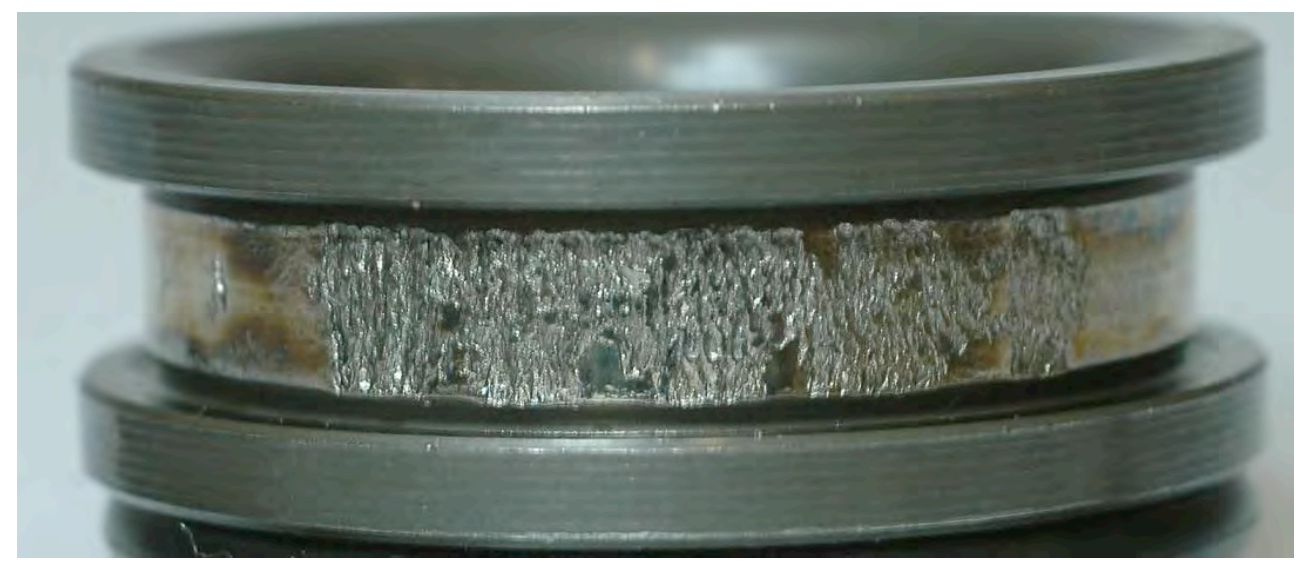

Bild 7: Geschälte Innenringlaufbahn des geprüften Planetenradlagers.

Betrachtet man die Änderung der Leitfähigkeit $\kappa$ über den Verlauf der Verlustleistung im Getriebe, so ergibt sich der in Bild8dargestellte Zusammenhang. Sowohl die steigende Änderung der Ölleitfähigkeit als auch die Getriebeverlustleistung sind mit dem Lagerverschleiß korreliert. 


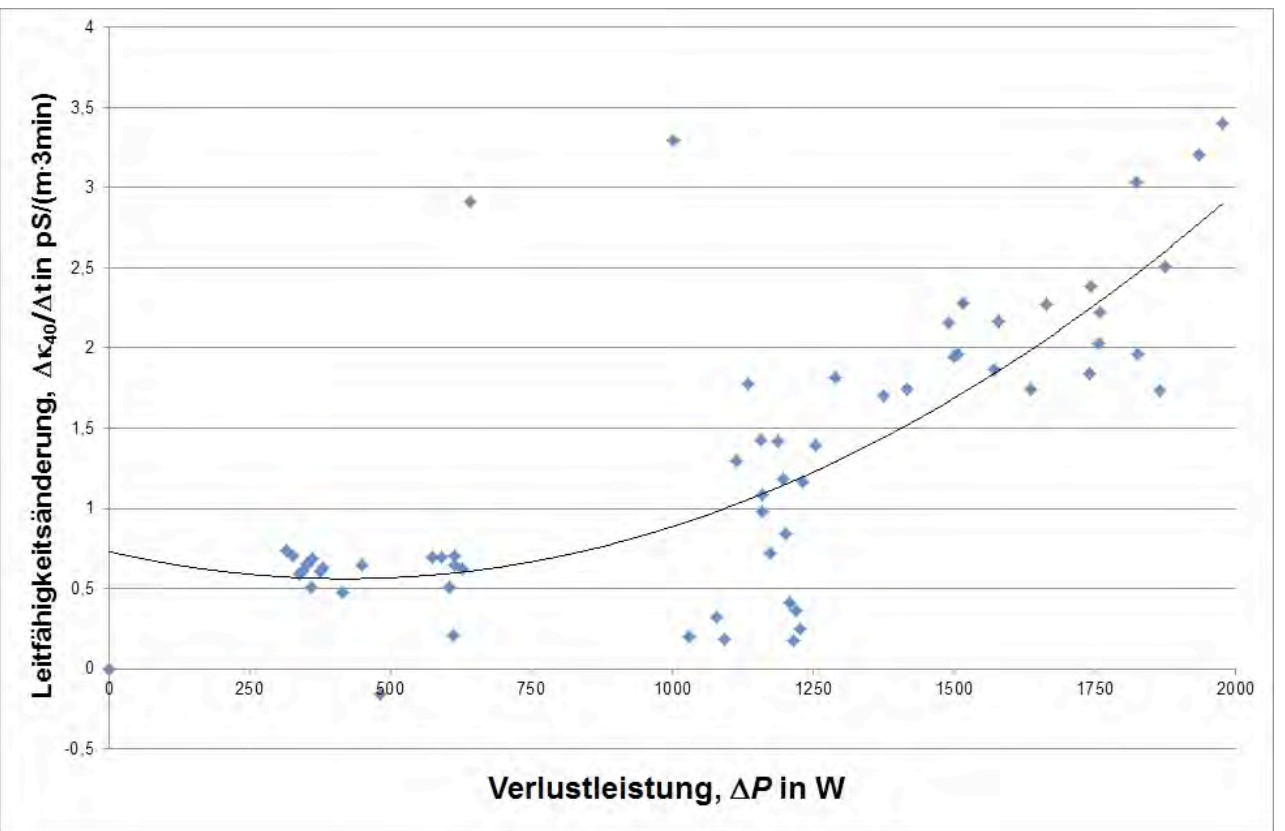

Bild8: Leitfähigkeitsänderung $\Delta \kappa_{40} / \Delta t$ in Abhängigkeit der Getriebeverlustleistung

Im Diagramm ist eine Trendlinie als Polynom dritten Grades eingezeichnet. Je größer die Steigungen der Leitfähigkeit und je größer die Verlustleistung im Getriebe desto größer ist auch der Lagerverschleiß. Außergewöhnliche Änderungen des Systems, wie z.B. die Umschaltung der Lastbedingungen sind nicht berücksichtigt.

\subsection{Dielektrizitätszahl des Getriebeöls}

Zusätzlich zur elektrischen Leitfähigkeit wird die Dielektrizitätszahl $\varepsilon_{\mathrm{r}}$ des Öls gemessen. Bei nicht additivierten Ölen lässt sich damit der Wassergehalt bestimmen. Es besteht Aussicht, dass die nachlassende Wirksamkeit von Additiven über die Dielektrizitätszahlmessung detektiert werden kann.

Bild 9 zeigt den schematischen Verlauf der temeraturkompensierte Dielektritzitätszahl $\varepsilon_{r 40}$ für additiviertes Öl bei Belastung mit Fremdstoffen wie Abrieb, Wasser und Säuren wie sie bei der Ölalterung entstehen. Beim Verbrauch der Additive fällt die Kurve, nach dem Verbrauch der Additive kommt es zum Wiederanstieg der Kurve. 


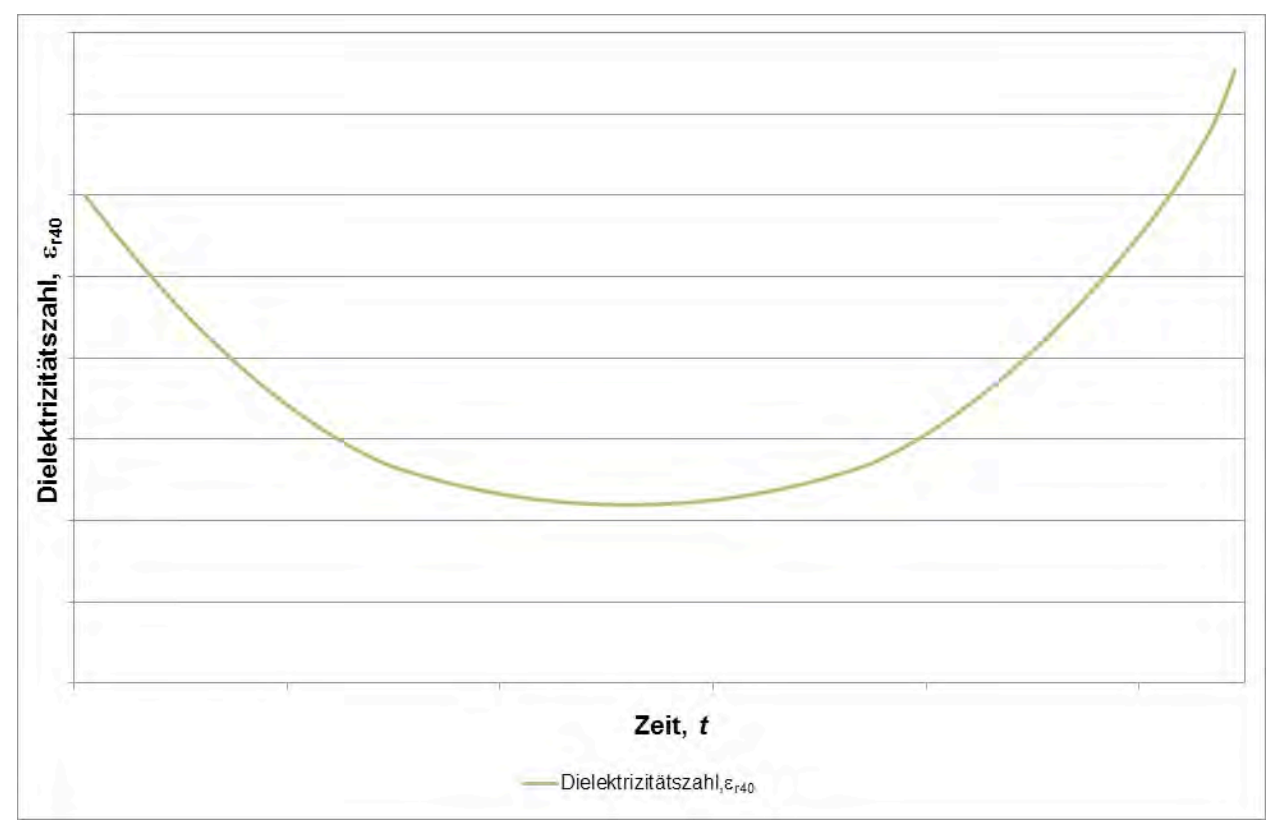

Bild9: Zeitlicher Verlauf der temperaturkompensierten Dielektrizitätszahl $\varepsilon_{\mathrm{r} 40}$.

\subsection{Untersuchung von Ölproben}

Vor dem Versuch, 30 Minuten vor Versuchsende und nach dem Versuch werden dem Prüfgetriebe Ölproben für eine Laboranalyse entnommen. Die durchgeführten Messungen umfassen die Bestimmung von Abriebkomponenten, Verschleißpartikeln und Verunreinigungen nach DIN 51396-1, die Änderung der Viskosität nach DIN 51562-1 und die Ermittlung des Viskositätsindex nach DIN ISO 2909. Zudem wird eine Messung der Neutralisationszahl (Säurezahl) nach DIN 51558-1 durchgeführt.

Die Befunde der drei entnommenen Ölproben zeigen im direkten Vergleich keine Abweichungen der analysierten Werte, die auf die massive Laufbahnschädigung des Planetenradlagers hindeuten. Die ermittelten sehr kleinen Abweichungen der analysierten Werte der drei Proben im Vergleich liegen innerhalb der Messtoleranzen des Analyseverfahrens zur Bestimmung der Abriebkomponenten, Verschleißpartikel etc.Dies ist ursächlich auf die hohe Qualität der Öle zurückzuführen und zeigt das solche massiven Schäden nicht unbedingt durch eine Partikel- oder Ölanalyse detektiertwerden können.

\section{Web-basiertes dezentrales Ölqualität Monitoring System}

Ein Web-basiertes, dezentrales Monitoringsystem, bei dem die Sensordaten vorzugsweise über das Internet übertragen und in eine als Bedienoberfläche des Monitoringsystems zu realisierende HTML-Seite eingetragen werden. Über die im Ölsensorsystem eingebauten LAN oder WLAN Schnittstellen können die Sensorsignale über das Internet an den Datenbankserver übertragen werden, der die Bedienoberfläche des Monitoringsystems als HTML-Seite für den autorisierten Bediener zur Verfügung stellt. Eine automatisierte Generierung von E-Mails, SMS usw. ist im Falle von Alarmmeldungen von jedem beliebigen Rechner mit Internetzugang möglich.

Wenn die Ölqualitätssensoren an den Anlagen montiert sind, kann die Messdatenpräsentation und -auswertung an anderer Stelle erfolgen. Ein flexibles, dezentrales Monitoringsystem ermöglicht die Auswertung der Messsignale und Überwachung der Anlagen auch durch externe Dienstleister. Eine anwenderorientierte Dienstleistung der quantifizierten Bewertung der Veränderungen des Systems Öl-Maschine inklusive der Empfehlung daraus abzuleitender, vorbeugender Wartungsmaßnahmen entlastet die Anlagenbetreiber, erhöht die Sicherheit und spart Kosten.

Über eine im Kommunikationsmodul eingebauten LAN / WLAN Schnittstelle und einem Webserver ist es dem Benutzer möglich, die aktuellen Werte des OilQSens ${ }^{\circledR}$ über einen Webbrowser an zu sehen. 
Nach Aufruf der Website und einer Kennwort geschützen Authorisierung wird dem Benutzer die letzten $\mathrm{x}$ Werte des Ölqualitätssensor OilQSens ${ }^{\circledR}$ dargestellt. Es erfolgt eine Darstellung sowohl in tabellarischer als auch grafischer Form.Bei der grafischen Darstellung ist es möglich verschiedene Parameter auswerten zu lassen. Des Weiteren stehen dem Benutzer die 3 Sprachen Deutsch, Englisch und Fränzösisch zur Verfügung.

In Bild 10 ist der die Website mit Visualisierung der Daten in tabellarischer Form dargestellt.

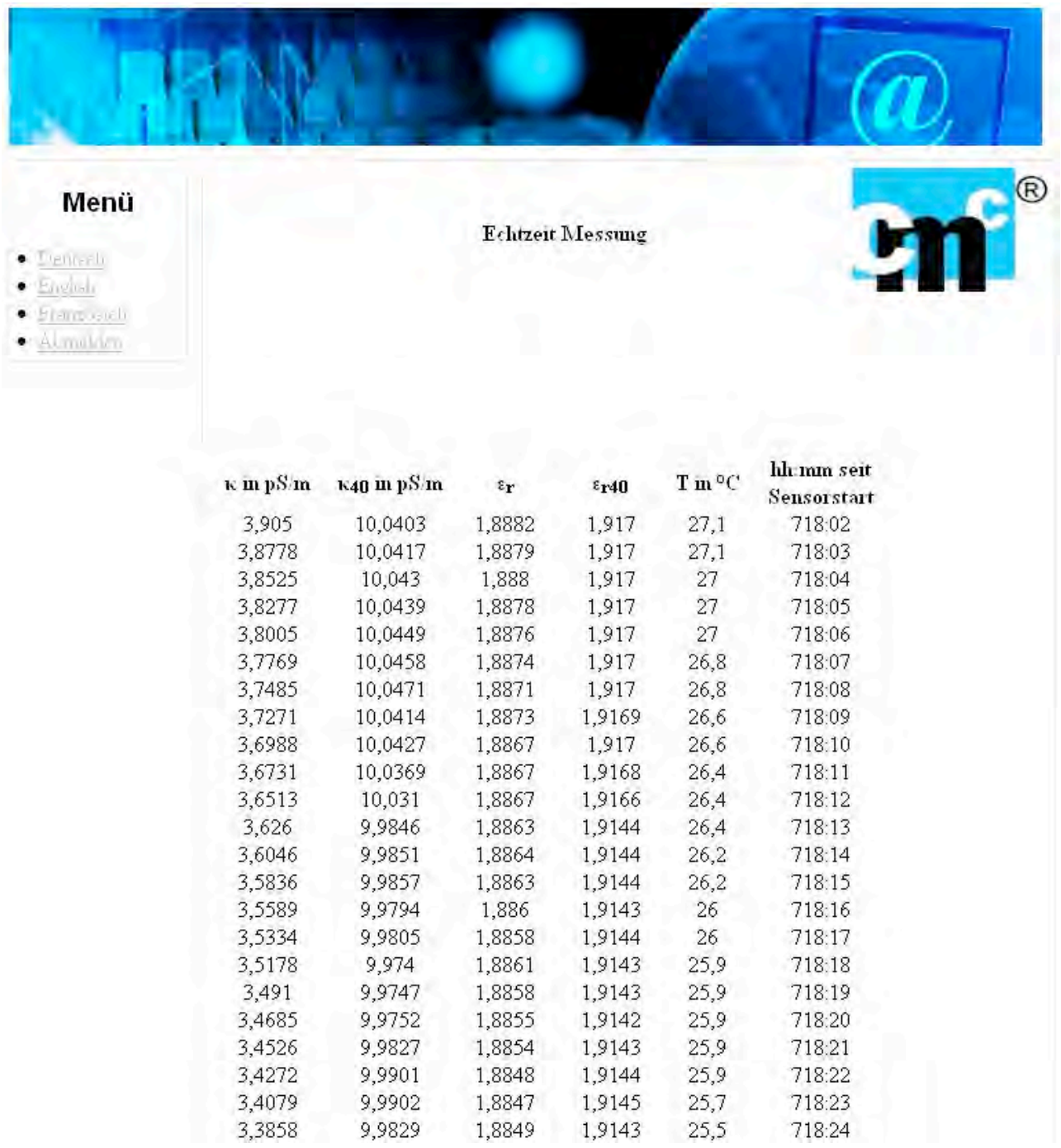

Bild 10: Realisierte HTML Seite des OilQSens ${ }^{\circledR}$ in tabellarischer Form

In Bild 11 ist Visualisierung von Daten in grafischer Form dargestellt. 


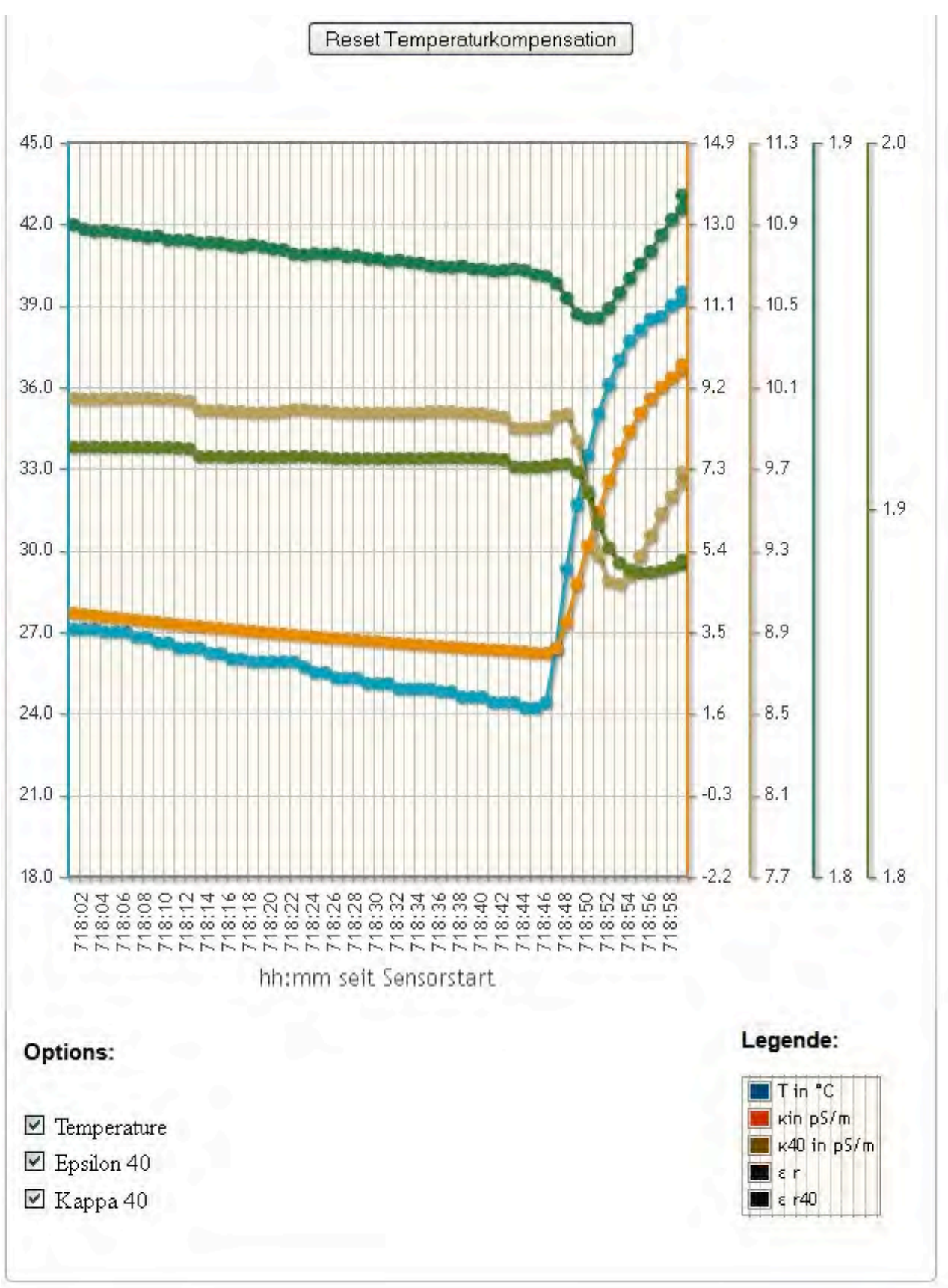

Bild 11: Realisierte HTML Seite des OilQSens ${ }^{\circledR}$ in grafischer Form

\section{Zusammenfassung}

Mit dem OilQSens ${ }^{\circledR}$ werden Komponenten der komplexen Impedanzen $\underline{X}$ der Öle, insbesondere die spezifischeelektrische Leitfähigkeit $\mathrm{k}$ und die Dielektrizitätszahl $\mathrm{e}_{\mathrm{r}}$ sowie die Öltemperatur T gemessen. Metallabrieb,gebrochene Ölmoleküle, Säuren oder Ölseifen bewirken eine Erhöhung der elektrischen Leitfähigkeit welche mit dem Verschmutzungsgrad direkt korreliert sind. Die dielektrischen Eigenschaften der Öle werden insbesondere durch den Wassergehalt bestimmt, der deshalb bei nicht additivierten Produkten ebenfalls über eine zusätzliche genaue Messung der Dielektrizitätszahl zugänglich wird. Bei additivierten Ölen lassen sich aus Dielektrizitätszahländerungen auch Aussagen über den Abbau von Additiven ableiten. Für eine effiziente Maschinenausnutzung und treffsichere Schadensverhütung besteht die Möglichkeit, vorbeugende Wartungsmaßnahmen zeitgerecht und bedarfsabhängig durchzuführen. Verschmutzungsgrad, Ölalterung und Versauerung, Wassergehalt bzw. Zersetzungszustand von 
Additiven bilden ein aussagefähiges Maß der Schmierstoff- und Werkstoffbeanspruchung für die onlineBetriebsüberwachung.

Das neu entwickelte Sensorsystem OilQSens ${ }^{\circledR}$ ermöglicht die Messung integraler Ölverschmutzung und deren zeitlicher Veränderung. Daraus kann auf den momentanen Bauelemente- und Geräteverschleiß geschlossen werden. Über LAN, WLAN oder serielle Schnittstellen des Sensorsystems werden die Messsignale in ein Web-basiertes Condition Monitoring System übertragen. Lange vor vorbeugenden Instandsetzungen können so vorbeugende, zustandsorientierte Wartungen indiziert werden.

Das Sensorsystem ermöglicht zudem eine quasi kontinuierliche Messung des Biodieselanteils in Diesel. Dieses kann zur Optimierung der Motorsteuerung in Dieselfahrzeugen, zukünftig voraussichtlich auch für mit Bioethanol / Benzin-Mischungen fahrende Flex-Fuel-Fahrzeuge genutzt werden.Das Bild 12 zeigt das OilQSens ${ }^{\circledR}$ Sensorsystem, bestehend aus Basissensor und Kommunikationsmodul.

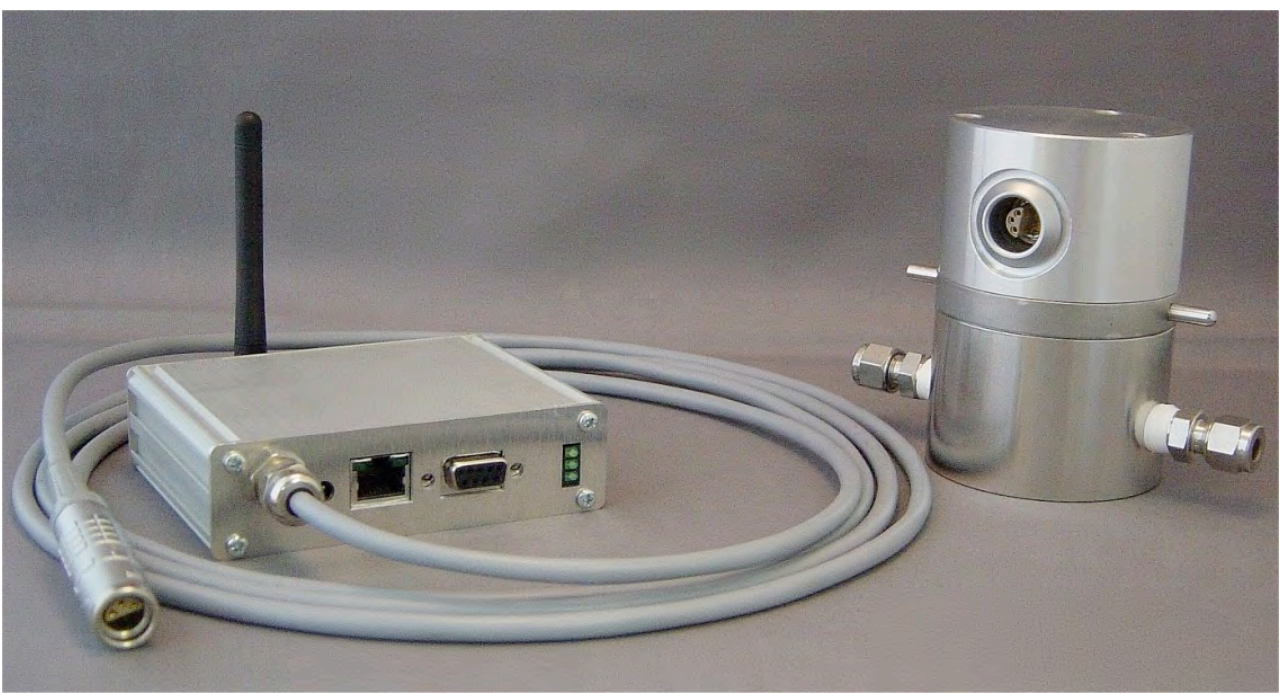

Bild 12: OilQSens ${ }^{\circledR}$ Sensorsystem mit Basissensor und Kommunikationseinheit

\section{Literatur:}

[1] E. Jochem, H. Bradke, C. Dreher, V. Ott, K. Alberti, J. Gerber, R. Masantschek, M. Ostwald, K. Kristof, J. Acosta, R. Bleischwitz, S. Bringezu und T. Ritthoff: Studie zur Konzeption eines Programms für die Steigerung der Materialeffizienz in mittelständischen Unternehmen. Anlagenband zur Potential- und Hemmnis-Analyse. Fraunhofer-Institut für System und Innovationsforschung (Fh-ISI), Arthur D. Little $\mathrm{GmbH}$, Wuppertal-Institut für Klima, Umwelt, Energie (WI), Wiesbaden 2005.

[2] J. Gegner und W. Nierlich: Operational Residual Stress Formation in Vibration-Loaded Rolling Contact. Advances in X-ray Analysis 52 (2008) S. 722-731.

[3] Kuipers, U., Mauntz, M.: Ölsensorsystem - Sensorsystem zur Messung von Komponenten der komplexen Impedanz elektrisch gering leitender und nichtleitender Fluide, dessen Realisierung und Anwendung, Patentanmeldung Nr. 102008047 366.9, Deutsches Patentamt, München, 2008

[4] Kuipers, U., Mauntz, M.: Verfahren, Schaltungsanordnung, Sensor zur Messung physikalischer Größen in Fluiden sowie deren Verwendung, Patentanmeldung Nr. 102008047 366.9, europäische Patentanmeldung, Europäisches Patentamt, München, 2008

[5]Bruggeman, D. A. G.: Berechnung verschiedener physikalischer Konstanten von heterogenen Substanzen 1. Dielektrizitätskonstanten und Leitfähigkeiten der Mischkörper aus isotropen Substanzen, Annalen der Physik, 5 Folge, Band 34, 1935 
[6]Gegner, J., Nierlich, W.: Mechanical and tribochemical mechanisms of mixed friction induced surface failures of rolling bearings and modeling of competing shear and tensile stress controlled damage initiation, International Conference FAILURES OF ROLLING BEARING BEARINGS, Bratislava 2010

[7] Gegner, J.,Hörz, G., Kirchheim, R.: Hydrogen Interaction with 0-, 1-, and 2-Dimensional Defects. In: A. W. Thompson, N. R. Moody (Hrsg.): Hydrogen Effects in Materials. ISBN: 0-87339-334-1, TMS The Minerals, Metals and Materials Society, Warrendale/Pennsylvania (1996) S. 35-46.

[8] Gegner, J., Nierlich, W.: Operational Residual Stress Formation in Vibration-loaded Rolling Contact. Advances in X-ray Analysis 52 (2008) S. 722-731.

[9] Gegner, J., Nierlich, W.: Einführung der Normalspannungshypothese für Mischreibung im WälzGleitkontakt, VDI Tagung mit Fachausstellung GLEIT- UND WÄLZLAGERUNGEN, Schweinfurt ,14.-15. Mai 2011

[10] Nierlich, W., Gegner, J.: Material Response Analysis of Rolling Bearings Using X-ray Diffraction Measurements. In: Proceedings of the Materials Week 2001, $4^{\text {th }}$ International Congress on Advanced Materials, their Processes and Applications [CD-ROM, ISBN 3-88355-302-6], $1^{\text {st }}-4^{\text {th }}$ October 2001, ICMInternational Congress Centre Munich. Werkstoffwoche-Partnerschaft (Hrsg.), Frankfurt, 2002

[11] Gegner, J.: Materialbeanspruchungsanalyse und ihre Anwendung auf Prüfstandsversuche zum Oberflächenausfall (Nierlich-Schadensmodus) von Wälzlagern. Materialwissenschaft und Werkstofftechnik 37 (2006) 3, S. 249-259.

[12] Nierlich, W., Gegner, J., Brückner, M.: XRD Residual Stress Analysis for the Clarification of Failure Modes of Rolling Bearings. Härterei-Technische Mitteilungen 62 (2007) 1, 27-31. 